\title{
Spectral Overlap Dependence of Enhanced Energy Transfer Near Small Au Nanoparticles
}

\author{
Cristian A. Marocico, Xia Zhang and A. Louise Bradley \\ School of Physics and CRANN, Trinity College Dublin, \\ College Green 2, Dublin 1, Dublin, Ireland \\ e-mail:bradlel@tcd.ie
}

\begin{abstract}
We consider the effect of gold nanospheres of subwavelength dimension on the energy transfer rates and efficiencies between donor-acceptor pairs placed near the nanospheres. We investigate theoretically the effect of the donor-Au NP and acceptor-Au NP separation on the energy transfer rates and efficiencies and the influence of the spectral overlap of the donor emission with the localised surface plasmon resonance.
\end{abstract}

Keywords: FRET, localized surface plasmons, Au nanospheres.

\section{INTRODUCTION}

The ability to control the interaction between quantum systems such as fluorescent dyes and quantum dots at the nanoscale has important applications in light-harvesting and emitting devices, sensing and length measurements on the scale of tens of nanometers, to name just some applications.

The process of resonance energy transfer in a donor-acceptor pair is one of the main pathways by which energy transfer occurs at the nanoscale. It plays a key role for example in biology (photosynthesis) [1], nanophotonics (LEDs, nanolasers) and microscopy [2,3], etc. The process is determined by the dipole-dipole interaction between the donor and acceptor and, in the short distance limit, has a $d^{-6}$ dependence on the donoracceptor separation $d$, characteristic of the Coulomb coupling between two dipoles, known as the Förster resonance energy transfer (FRET) regime [4].

The Förster regime is typically valid below approximately $10 \mathrm{~nm}$ above which the energy transfer process is overwhelmed by other de-excitation mechanisms, such as phonon relaxation or emission into the far-field. In a variety of applications, it is desirable to extend the FRET regime to larger donor-acceptor separations. This could potentially be used to enhance the efficiency of light-harvesting [5,6] and emitting systems, as well as increase the range and accuracy of sensing devices.

One possible way of enhancing the energy transfer rate in a donor-acceptor pair is by introducing a "mediator". The role of the mediator is to increase the probability that the energy transfer between donor and acceptor will occur. An example of such a mediator is a metal nanoparticle. Metal nanoparticles can support localized surface plasmons (LSPs), whose main characteristics are a large confinement and a large enhancement of the electromagnetic field. The field of a LSP thus provides a good channel for the energy transfer process between donor and acceptor when these are suitably placed around the nanoparticle $[7,8]$.

The field of the LSP on the metal nanoparticle influences not only the energy transfer rate between donor and acceptor, but also the other channels which contribute to the total decay rate of the donor and introduces new decay channels, such as absorption of energy by the metal. It is therefore important to enhance not only the energy transfer rate, but in particular the energy transfer efficiency, which is a measure of the competition between the donor-acceptor energy transfer rate and all other decay channels of the donor.

In this contribution we investigate the role of the LSP on an Au nanoparticle ( $\mathrm{Au} N \mathrm{NP}$ ) in modifying the energy transfer rate and efficiency of a donor-acceptor pair. In particular we consider an Au NP with a small diameter $(5.0 \mathrm{~nm})$ and donor-acceptor pairs with optical properties close to those of quantum dots used in reported experimental work [9]. Specifically we probe the influence of the spectral overlap of the donor emission with the LSP resonance on the energy transfer properties. It was previously experimentally reported that LSP enhanced FRET was very sensitive to the donor emission properties [7].

Within a Green's tensor formalism, we have investigated the dependence of the energy transfer efficiency on the donor-Au NP and acceptor-Au NP distance and donor-LSP spectral overlap for two donor-acceptor pairs.

\section{THEORETICAL FRAMEWORK}

Using a Green's tensor formalism, which we briefly sketch below, we can calculate the energy transfer rate and efficiency between two point dipoles placed near a metal nanoparticle.

The Green's tensor of a particular arrangement of material bodies represents the electromagnetic response of that arrangement to a point dipole excitation. Its components are, in essence, the components of the electric field produced by a point dipole oriented along three orthogonal axes.

It can be shown that the energy transfer (ET) rate between a donor-acceptor pair, modeled as point dipoles, is given as [9] 


$$
k_{\mathrm{ET}}=\frac{36 \pi^{2} Y_{D}}{\tau_{\mathrm{D}}} \int_{0}^{\infty} \frac{\mathrm{d} \lambda}{\lambda^{2}} f_{\mathrm{D}}(\lambda)\left|\mathbf{n}_{\mathrm{A}} \cdot \mathfrak{G}\left(\mathbf{r}_{\mathrm{A}}, \mathbf{r}_{\mathrm{D}}, \lambda\right) \cdot \mathbf{n}_{\mathrm{D}}\right|^{2} \sigma_{\mathrm{A}}(\lambda) .
$$

where $\tau_{\mathrm{D}}$ is the donor lifetime in the absence of the acceptor, $Y_{\mathrm{D}}$ is the quantum yield of the donor, $f_{\mathrm{D}}(\lambda)$ is the area-normalized donor emission spectrum, i.e. $\int_{0}^{\infty} \mathrm{d} \lambda f_{\mathrm{D}}(\lambda)=1, \sigma_{\mathrm{A}}$ is the acceptor absorption cross-section and $\mathfrak{G}\left(\mathbf{r}_{\mathrm{A}}, \mathbf{r}_{\mathrm{B}}, \lambda\right)$ is the Green's tensor in the particular geometry. Finally, $\mathbf{n}_{\mathrm{D}(\mathrm{A})}$ is a unit vector along the direction of the transition dipole moment of the donor (acceptor).

The energy transfer process from the donor to the acceptor competes with the decay process of the donor by other means, e.g. emission into the far-field, phonon relaxation, losses in the metal NP. The rate of this process is encapsulated in the decay rate $k_{\mathrm{SE}}$, which can also be calculated via the Green's tensor as

$$
k_{\mathrm{SE}}=\int_{0}^{\infty} \mathrm{d} \lambda f_{\mathrm{D}}(\lambda) \gamma(\lambda)
$$

where $\gamma(\lambda)$ is the single-frequency decay rate, given as

$$
\gamma(\mathbf{r}, \omega)=\frac{2 \omega^{2} \mu^{2}}{\hbar \varepsilon_{0} c^{2}} \operatorname{Im}\left[\mathbf{n}_{\mu} \cdot \mathfrak{G}(\mathbf{r}, \mathbf{r}, \omega) \cdot \mathbf{n}_{\mu}\right]
$$

with $\mu$ the transition dipole moment of the emitter and $\mathbf{n}_{\mu}$ a unit vector in the direction of this dipole moment.

In the absence of any material bodies in their vicinity, and when the donor-acceptor distance is much smaller than the donor emission wavelength, the energy transfer process is in the Förster regime and the above expression for the ET rate simplifies to [10]

$$
k_{\mathrm{ET}}=\frac{1}{\tau_{\mathrm{D}}}\left(\frac{R_{0}}{r}\right)^{6}
$$

where $R_{0}$ is the Förster radius, representing the distance at which the energy transfer rate is equal to all the other decay channels of the donor. The Förster radius can be calculated from the overlap integral between the donor emission spectrum, $f_{\mathrm{D}}(\lambda)$, and the acceptor absorption cross-section, $\sigma_{\mathrm{A}}(\lambda)$ as

$$
R_{0}=\left[\frac{3 c}{32 \pi^{4} n^{4}} \int_{0}^{\infty} \mathrm{d} \lambda \lambda^{2} f_{\mathrm{D}}(\lambda) \sigma_{\mathrm{A}}(\lambda)\right]^{1 / 6}
$$

With the decay and energy transfer rates defined, one can introduce an energy transfer efficiency defined as

$$
\eta_{\mathrm{ET}}=\frac{k_{\mathrm{ET}}}{k_{\mathrm{SE}}+k_{\mathrm{ET}}}
$$

to quantify the competition between the energy transfer process from donor to acceptor and all other decay channels of the donor. In the Förster regime, the energy transfer efficiency has the form

$$
\eta_{\mathrm{ET}}=\frac{1}{1+\left(\frac{r}{R_{0}}\right)^{6}}
$$

and the Förster radius is seen to be the donor-acceptor separation at which $\eta=50 \%$.

In the presence of material bodies, the energy transfer rate no longer simplifies to Eq. (4) and one must use the full expression (1) to calculate the rate. The energy transfer efficiency can still be calculated via Eq. (2.6), but, in general, one does not expect the distance dependence to be $r^{-6}$. This model has been previously validated by comparison with experimental results reported in reference [9].

\section{RESULTS AND DISCUSSION}

\subsection{Optical Properties}

In this contribution we will consider an Au NP with a diameter of $D=5 \mathrm{~nm}$. The extinction, absorption and scattering efficiencies for this Au NP are presented in Fig. 1a. The LSP supported by the Au NP appears as a peak in the extinction and absorption efficiencies. The LSP peak is centred at $500 \mathrm{~nm}$, and the broadening of the peak is caused by surface scattering effects, which can be considerable for particles much smaller than the mean-free path of electrons in the metal $(\sim 50 \mathrm{~nm}$ for $\mathrm{Au})$.

Figure $1 \mathrm{~b}$ shows the field intensity of a plane wave scattering off of the Au NP, with its characteristic dipole pattern. The wavelength of the incoming plane wave is $\lambda=550 \mathrm{~nm}$, close to the LSP peak of the Au NP.

Finally, Fig. 1c shows the emission spectrum of two donors, the first centred at $\lambda_{\mathrm{D}}=500 \mathrm{~nm}$, the second at $\lambda_{\mathrm{D}}=550 \mathrm{~nm}$, together with the absorption spectrum of the acceptor. The acceptor absorption spectrum is taken from experiments and typical of a quantum dot acceptor Ref. [9], scaled appropriately. The donor emission spectra represent Gaussian fits to the typical quantum dot experimental spectra for example see reference [11]. For comparison, we also show the calculated Au NP extinction spectrum. 


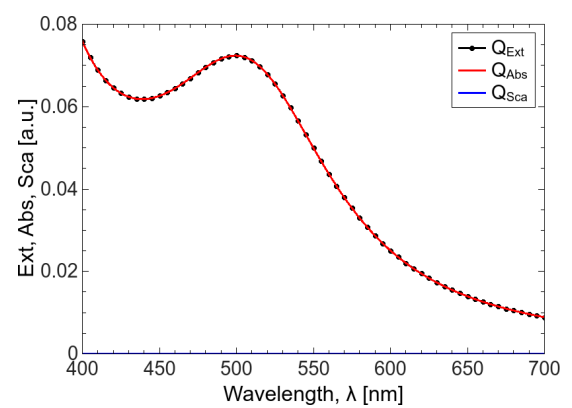

(a)

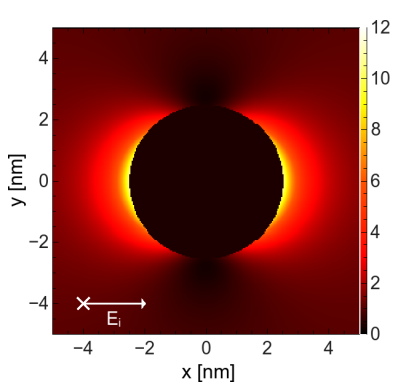

(b)

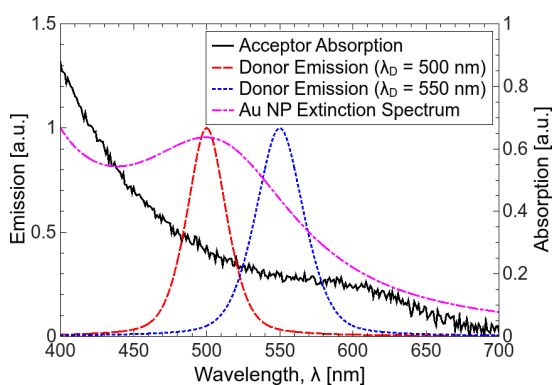

(c)

Figure 1: (a) Extinction, scattering and absorption efficiencies of an Au NP with a diameter D = $5 \mathrm{~nm}$; (b) Field profile of a plane wave scattering of an Au NP; (c) Emission and absorption profiles of the donors and acceptor.

\subsection{Energy Transfer Rates}

We consider the energy transfer rates between a donor and an acceptor separated by the Au NP for two donoracceptor pairs, with the donor emission having a different spectral overlap with the surface plasmon resonance. In each case we investigate when either the donor or the acceptor position is kept fixed while the position of the other member of the pair is varied. Figure 2 shows the energy transfer rate for the donor acceptor pair as a function of their separation, for two donor emission wavelengths and two donor distances to the surface of the $\mathrm{Au}$ NP.

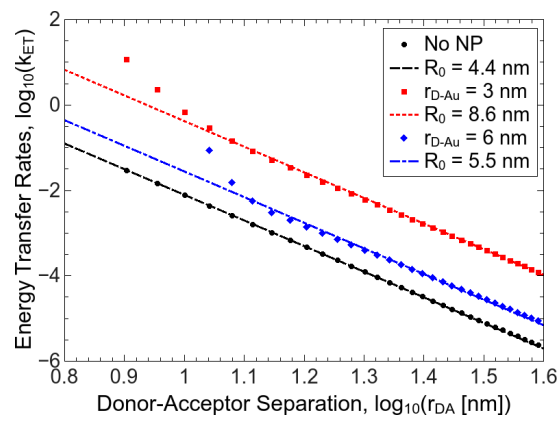

(a)

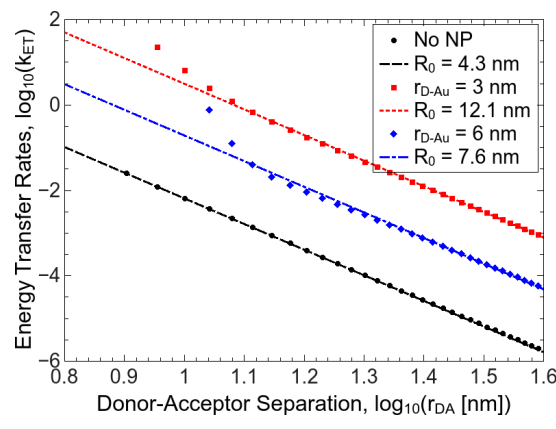

(b)

Figure 2. Energy transfer rates for several donor positions and donor emission wavelengths of (a) $\lambda=500 \mathrm{~nm}$ and (b) $\lambda=550 \mathrm{~nm}$.

The symbols are the numerical calculations, whereas the lines constitute fits with a Förster model, from which the Förster radius is extracted. In panel (a) the donor emission wavelength is $\lambda=500 \mathrm{~nm}$, while in panel (b) it is $\lambda=550 \mathrm{~nm}$. For both wavelengths, the Förster radius is increased in the presence of the Au NP. The increased Förster radius is evident for both donor distances to the surface of the Au NP, $r_{\mathrm{D}}=3 \mathrm{~nm}$ and $6 \mathrm{~nm}$. The enhancement of the Förster radius is larger, however, for panel (b), when the emission wavelength is $550 \mathrm{~nm}$. It can be noted that the Förster radii for both donor-acceptor pairs are similar with values of $4.4 \mathrm{~nm}$ and $4.3 \mathrm{~nm}$ in the absence of the Au NP. Therefore the increased Förster radius for a donor emitting at $550 \mathrm{~nm}$ is a consequence of the influence of the spectral overlap between the donor emission and the LSP resonance.

If we consider a fixed position of the acceptor and allow the donor position to vary, as depicted in Fig. 3, the influence of the Au NP is seen to rapidly diminish with increasing distance of the donor to the surface of the NP. There is still a slight enhancement of the Förster radius as extracted from the fit, but it is much smaller.

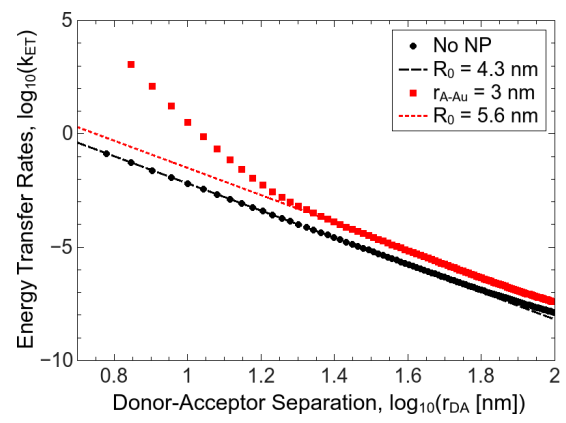

Figure 3. Energy transfer rates for $r_{A-A u}=3 \mathrm{~nm}$ and donor emission wavelength of $\lambda=550 \mathrm{~nm}$. 


\section{CONCLUSIONS}

We have considered the effect of a small Au NP, with a diameter of $5 \mathrm{~nm}$, on the energy transfer rate between a donor-acceptor pair placed on opposite sides of the NP. Two donor-acceptors pairs have been studied to investigate the influence of the spectral overlap of the donor emission with the LSP resonance on the energy transfer rate. The two pairs have the same acceptor. For both pairs it is observed that the donor-Au NP coupling is by far the strongest whereas the acceptor-Au NP coupling is much weaker.

The enhancement of the Förster radius of the donor-acceptor pairs can be attributed to the LSP excited by the donor at the surface of the Au NP. For a fixed donor-Au NP separation, the energy transfer predominantly follows a FRET trend, with a $r^{-6}$ distance dependence. The coupled donor-Au NP pair behaves as an enhanced donor dipole. In all cases at the smallest donor-acceptor separations the energy transfer rate is greater than predicted by the FRET fit. For a fixed acceptor-Au NP separation as the donor is moved away from the NP the energy transfer rate rapidly reverts to close to its value in the absence of the Au NP, and again a $r^{-6}$ distance dependence is recovered. It is seen that the enhancement of the energy transfer rate and Förster radius is strongly dependent on the spectral overlap of the donor emission with the LSP resonance. Both donor-acceptor pairs have similar Förster radii in the absence of the Au NP. Larger enhancement of the energy transfer rate and Förster radius is observed when the donor emission peak is red-shifted with respect to the LSP resonance wavelength at the peak of the far-field extinction spectrum.

The large increase in the effective range of the energy transfer process by the introduction of the Au NP can have important applications in light harvesting and light emitting devices, as well as optical rulers.

\section{ACKNOWLEDGEMENTS}

This work was supported by the Science Foundation Ireland under grant No. 10/IN.1/12975.

\section{REFERENCES}

[1] D. L. Andrews and A.A. Demidov, editors, Resonance Energy Transfer (Wiley, New York, 1999).

[2] J. Z. Zhang and C. Noguez, Plasmonics 3, 127 (2008).

[3] F. De Angelis, M. Malerba, M. Patrini, E. Miele, G. Das, A. Toma, R.P. Zaccaria, and E. Di Fabrizio, Nano Lett. 13, 3553 (2013).

[4] T. Förster, Ann. Phys. 437, 55 (1948).

[5] M. Law, L.E. Greene, J.C. Johnson, R. Saykally, and P. Yang, Nat. Mater. 4, 455 (2005).

[6] O.L. Muskens, J.G. Rivas, R.E. Algra, E.P.A.M. Bakkers, and A. Lagendijk, Nano Lett. 8, 2638 (2008).

[7] M. Lunz, V.A. Gerard, Y.K. Gun'ko, V. Lesnyak, N. Gaponik, A.S. Susha, A.L. Rogach, and A.L. Bradley, Nano Lett. 11, 3341 (2011).

[8] J. Zhang, Y. Fu, and J.R. Lakowicz, J. Phys. Chem. C. Nanomater. Interfaces 111, 50 (2007).

[9] X. Zhang, C.A. Marocico, M. Lunz, V.A. Gerard, Y.K. Gun'ko, V. Lesnyak, N. Gaponik, A.S. Susha, A.L. Rogach, and A.L. Bradley, ACS Nano 8, 1273 (2014).

[10] L. Novotny and B. Hecht, Principles of Nano-Optics, 2nd ed. (Cambridge University Press, Cambridge, UK, 2012).

[11] X. Zhang, C.A. Marocico, M. Lunz, V.A. Gerard, Y. K. Gun'ko, V. Lesnyak, N. Gaponik, A.S. Susha, A.L. Rogach, and A.L. Bradley, ACS Nano 6, 9283 (2012). 